\title{
Post-Mortem 7.0-Tesla Magnetic Resonance Imaging of the Brain in a Patient with Disseminated Intravascular Coagulation
}

\author{
Jacques De Reuck*
}

INSERM 1171 "Degenerative and Vascular Cognitive Disorders," Université de Lille 2, France

*Corresponding Author: Jacques De Reuck, Ryvissche Park 16, 9052 Zwijnaarde, Belgium.

\section{ABSTRACT}

A post-mortem 7.0-tesla MRI study of the brain in a patient with disseminated intravascular coagulopathy is presented.

\section{INTRODUCTION}

Disseminated intravascular coagulation (DIC) associated with multiple organ dysfunctions plays a pivotal role in severe sepsis [1]. No postmortem MRI findings of the brain lesions are available in DIC.

\section{RESULTS}

We present a post-mortem 7.0-tesla MRI study of the brain in a 31-year woman with an acute sepsis complicated by DIC. Six coronal sections of the left cerebral hemisphere were examined with $\mathrm{T} 2$ and $\mathrm{T} 2 *$ sequences.

On the T2 sequence a medial temporal lobar haematoma was observed. No ischemic lesions could be detected (Fig.1). In addition to the haematoma several small bleeds were observed on the $\mathrm{T} 2 *$ sequence in the cerebral cortex of all 6 coronal sections. Also a hemorrhage in the centrum semiovale was present on one section (Fig. 2).

\section{Fig. 1}
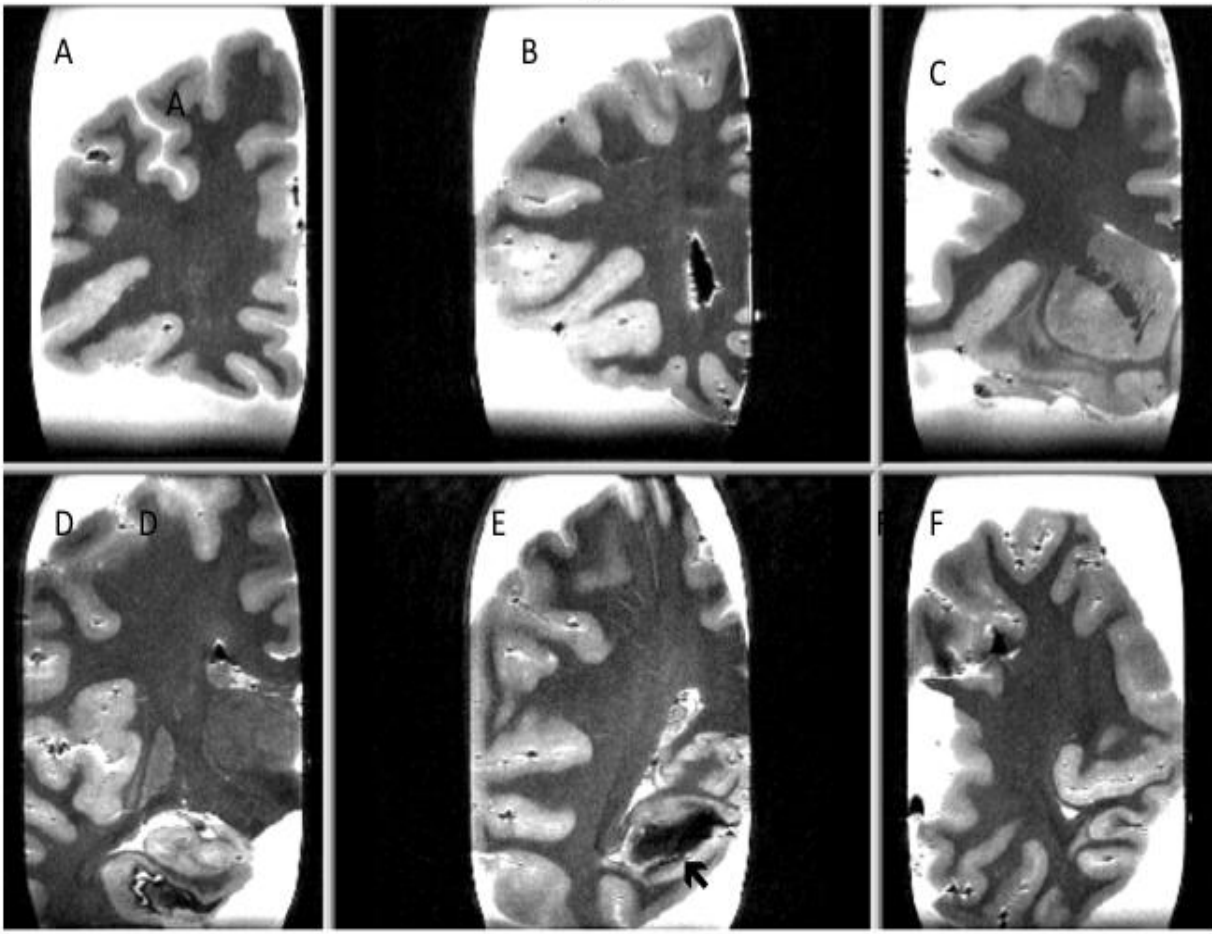


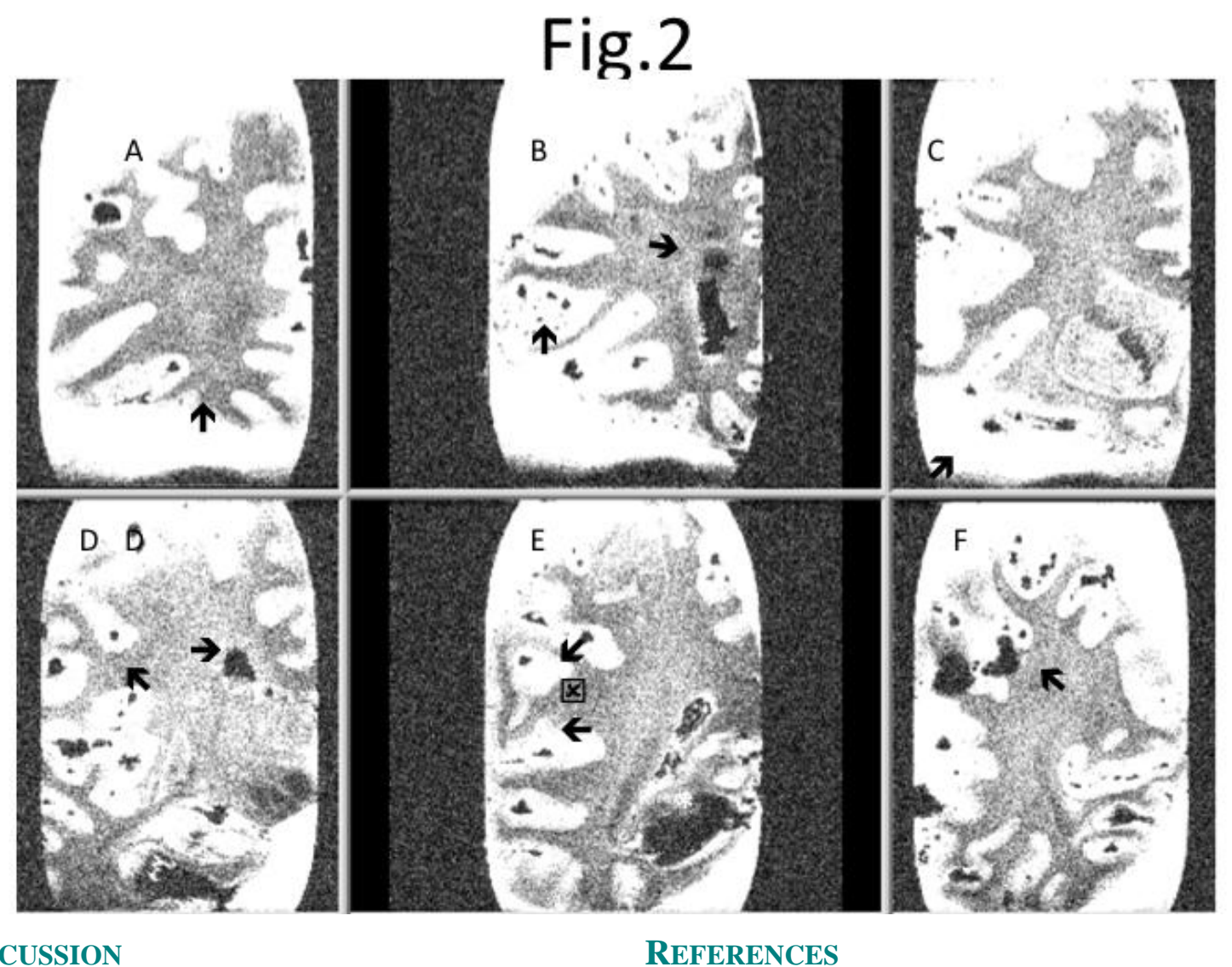

\section{Discussion}

The present post-mortem MRI of a patient with DIC shows only haemorrhages without additional ischemic lesions. These findings confirm some observations in still living patients [2,3].

\section{DISCLOSURES}

The author has nothing to declare in relation to this article. No funding was received for the publication of this article.

\section{REFERENCES}

[1] Cando S, Shiraishi A, Yamakawa K, et al. Role of disseminated intravascular coagulation in severe sepsis. Thromb Res. 2019; 178: 182-188.

[2] Neligan A, Rajakuledran S, Nortley R and Manji AL. Extensive cerebral micro hemorrhages caused by acute disseminated intravascular coagulation secondary to sepsis. JAMA Neurol. 2014; 71 (4): 510-511.

[3] Baeck HJ, Lee DH, Han KH, et al. Fatal intracranial hemorrhage in a patient with intravascular coagulation associated with sepsis. Korean J Critic Care Med. 2016; 31(2): 134-139.

Citation: Jacques De Reuck, "Post-Mortem 7.0-Tesla Magnetic Resonance Imaging of the Brain in a Patient with Disseminated Intravascular Coagulation", International Journal of Research Studies in Medical and Health Sciences. 2020; 5(10): 1-2.

Copyright: () 2020 Jacques De Reuck, This is an open-access article distributed under the terms of the Creative Commons Attribution License, which permits unrestricted use, distribution, and reproduction in any medium, provided the original author and source are credited. 